\title{
CONSTRAINED CONTROL AND RATE OR INCREMENT FOR LINEAR SYSTEMS WITH ADDITIVE DISTURBANCES
}

\author{
FOUAD MESQUINE, FERNANDO TADEO, AND ABDELLAH BENZAOUIA
}

Received 17 May 2005; Revised 9 May 2006; Accepted 18 May 2006

This paper is devoted to the control of linear systems with constrained control and rate or increment with additive bounded disturbances. Necessary and sufficient conditions such that the system evolution respects rate or increment constraints are used to derive stabilizing feedback control. The control law respects both constraints on control and its rate or increment and is robust against additive bounded disturbances. An application to a surface mount robot, where the $Y$-axis of the machine uses a typical ball screw transmission driven by a DC motor to position circuits boards, is achieved.

Copyright (c) 2006 Fouad Mesquine et al. This is an open access article distributed under the Creative Commons Attribution License, which permits unrestricted use, distribution, and reproduction in any medium, provided the original work is properly cited.

\section{Introduction}

Usually, real or physical plants are subject to constrained variables. The most frequent constraints are of saturation type: limitations on the magnitude of certain variables. Hence, this topic is of continuing interest and one could find many approaches to study this problem; not exhaustively, the positive invariance concept $[4-6,8,10]$ (and the references therein) the small and high gain concept [21], the $l_{-} 1$ optimization concept [12], and so on, can be cited. Other types of rate or incremental constraints were introduced while considering predictive control and practical applications $[1,11,25]$, and other approaches $[14,25]$, and so forth.

In fact, for some processes, the rate of changes variable is limited within given bounds. These limits can arise from physical limits or from modelling assumptions, if exceeded, could damage the process or destroy limits of linear model validity. Most synthesis methods are based on symmetric constraints. However, in most real-life applications, the constraints are not symmetric, from economical and safety reasons. For example, in process control applications, the nominal working point of the valves is usually near the upper or lower limits, in mechanical systems, the maximum acceleration is usually smaller than the maximum deceleration (for safety reasons). In a previous work, the problem of

Hindawi Publishing Corporation

Mathematical Problems in Engineering

Volume 2006, Article ID 37591, Pages 1-16

DOI 10.1155/MPE/2006/37591 
nonsymmetrical constrained control and rate or increment was successfully addressed [23]. Henceforth, this work extends those results to the case of nonsymmetrical constrained systems with additive bounded disturbances and shows their applicability on a real process, namely, a surface mount robot. Necessary and sufficient conditions of positive invariance for incremental domains with respect to autonomous systems with additive bounded disturbances are then derived. Further, a link is done between a pole assignment procedure and these conditions to find stabilizing linear state feedback controllers respecting both nonsymmetrical constraints on control and rate with additive bounded disturbances.

It is worth noticing here that the problem of constrained control of linear systems can be seen as the problem of control of nonlinear systems where the saturation is treated as a locally sector bounded nonlinearity $[15,19]$. In these works and references therein, absolute stability analysis tools as circle and Popov criteria are used. Further, perturbation rejection as studied thereafter was also studied in $[8,9]$ using the set invariance concept and in [18] using convex writing of the saturation function.

In this last approach, saturations are allowed to take effect while guaranteeing asymptotic stability (see $[16,17]$ and the references therein). Allowing the control to saturate leads to a bounded region of stability that can be easily obtained by the resolution of a set of $2^{m}$ LMIs. But this set is ellipsoidal and symmetric which may be conservative. The main challenge in these approaches is to obtain a large enough domain of initial states which ensures asymptotic stability for the system despite the presence of saturations $[3,13,16]$. For all these previous works treating saturating control or constrained control, there is no constraints on the rate of the control. Hence, this work is the extension to those using the positive invariance concept to deal with rate and magnitude control constraints in the presence of additive bounded disturbances.

On the other hand, for the application, surface mount robots are of great interest in modern industry. This interest is justified by the presence of positioning systems in practically all industrial applications. In the prolific literature, many works about these systems classically controlled by motors can be found, [20] and references therein. Hence, stabilizing state feedback to an assembly machine for mounting electronic components is derived. The $Y$-axis of the machine uses a ball screw transmission driven by a current controlled DC motor that has constrained control and rate with additive bounded disturbances.

1.1. Notations. For a scalar $a \in \mathbb{R}$, we define $a^{+}=\sup (a, 0)$ and $a^{-}=\sup (-a, 0)$. For $x \in \mathbb{R}^{n}$, a vector $\delta x(t)$ denotes the derivative with respect to time for the continuous-time case and $x(t+1)$ for the discrete-time case. Further, we note $x^{+}=\left(x_{j}^{+}\right)$and $x^{-}=\left(x_{j}^{-}\right)$for $j=1, \ldots, n$. Furthermore, for a matrix $A=(a)_{i j}, i, j=1, \ldots, n$, the tilde transforms are defined by

$$
\tilde{A}=\left[\begin{array}{ll}
A^{+} & A^{-} \\
A^{-} & A^{+}
\end{array}\right],
$$


where $A^{+}=\left(a^{+}\right)_{i j}$ and $A^{-}=\left(a^{-}\right)_{i j}, i, j=1, \ldots, n$, and

$$
\widetilde{A_{c}}=\left[\begin{array}{ll}
A_{1} & A_{2} \\
A_{2} & A_{1}
\end{array}\right]
$$

where

$$
A_{1}=\left\{\begin{array}{l}
a_{i i}, \\
a_{i j}^{+} \quad \text { for } i \neq j,
\end{array} \quad A_{2}=\left\{\begin{array}{l}
0, \\
a_{i j}^{-} \quad \text { for } i \neq j .
\end{array}\right.\right.
$$

Also, $\sigma(A)$ denotes the spectrum of matrix $A$, $\square$ denotes the identity matrix of appropriate size, and $\mathbb{D}_{s}$ denotes the stability domain for eigenvalues (i.e., the left complex half-plane for the continuous-time case and the unity disk for the discrete-time case).

\section{Problem statement}

Consider the linear time-invariant system

$$
\delta x(t)=A x(t)+B u(t)+E p(t)
$$

where $x(t) \in \mathbb{R}^{n}$ is the state of the system, $u(t) \in \mathbb{R}^{m}$ is the input, constrained to evolve in the following domain:

$$
D_{u}=\left\{u(t) \in \mathbb{R}^{m},-u_{\min } \leq u(t) \leq u_{\max }, u_{\min }, u_{\max } \in \operatorname{Int} \mathbb{R}_{+}^{m}\right\}
$$

The control rate or increment is constrained as follows:

$$
\begin{gathered}
-\Delta_{\min } \leq \dot{u}(t) \leq \Delta_{\max }, \\
-\Delta_{\min } \leq u(t+1)-u(t) \leq \Delta_{\max },
\end{gathered}
$$

$p(t)$ is an additive disturbance bounded as

$$
-p_{\min } \leq p(t) \leq p_{\max }
$$

Further, we denote

$$
U=\left[\begin{array}{l}
u_{\max } \\
u_{\min }
\end{array}\right], \quad \Delta=\left[\begin{array}{c}
\Delta_{\max } \\
\Delta_{\min }
\end{array}\right], \quad P=\left[\begin{array}{c}
p_{\max } \\
p_{\min }
\end{array}\right] .
$$

The problem, to be solved, is to find stabilizing linear state feedback as

$$
u(t)=F x(t), \quad F \in \mathbb{R}^{m \times n},
$$

ensuring closed loop asymptotic stability of the system despite perturbations with no violation of nonsymmetrical constraints on the rate (or increment) and magnitude of the control. 
4 Constrained control and rate with disturbances

\section{Preliminaries}

The previously stated problem, without additive disturbances, was solved in [23]. This paper presents the extension of this problem to the case of systems with additive bounded disturbances as this is a frequent situation in practice. First, consider the linear time-invariant autonomous system with additive bounded disturbances given by

$$
\delta z(t)=H z(t)+E p(t), \quad z\left(t_{o}\right)=z_{o}
$$

where $z \in \mathbb{R}^{m}$ is the state, constrained to evolve in the domain

$$
D_{z}=\left\{z \in \mathbb{R}^{m},-z_{\min } \leq z(t) \leq z_{\max }, z_{\min }, z_{\max } \in \operatorname{Int} \mathbb{R}_{+}^{m}\right\}
$$

and $p(t)$ is the perturbation, bounded in the domain

$$
D_{P}=\left\{p(t) \in \mathbb{R}^{p},-p_{\min } \leq p(t) \leq p_{\max }, p_{\min }, p_{\max } \in \operatorname{Int} \mathbb{R}_{+}^{p}\right\} .
$$

Consider also that the rate (or increment) of the state vector is constrained as follows:

$$
\begin{gathered}
-\Delta_{\min } \leq \dot{z}(t) \leq \Delta_{\max }, \\
-\Delta_{\min } \leq z(t+1)-z(t) \leq \Delta_{\max } .
\end{gathered}
$$

Now, we recall the definition of $D_{P}$-positive invariance of domain $D_{z}$, which is very useful for the sequel.

Definition 3.1. Domain $D_{z}$ given by (3.2) is $D_{P}$-positively invariant with respect to motion of system (3.1) if for all initial conditions $z_{o} \in D_{z}$, the trajectory of the system $z\left(t, t_{o}, z_{o}\right) \in D_{z}$ for all $p(t) \in D_{P}, t>t_{o}$.

$D_{P}$-positive invariance conditions have already been reported in $[4,8]$ for the discretetime case and in [22] for the continuous-time case. Let us recall these conditions hereafter.

Theorem $3.2[4,8,22]$. Domain $D_{z}$ is $D_{P}$-positively invariant with respect to motion of the system with additive disturbances (3.1) if and only if matrix $H$ satisfies

$$
\begin{gathered}
\widetilde{H_{c}} Z+\widetilde{E} P \leq 0 \quad \text { for the continuous-time case, } \\
\tilde{H} Z+\widetilde{E} P \leq Z \text { for the discrete-time case. }
\end{gathered}
$$

Evolution of the autonomous system (3.1) will respect both constraints on the state $z(t)$ and constraints on its rate or increment if domain $D_{z}$ given by (3.2) is $D_{P}$-positively invariant and conditions established therafter in a technical lemma are satisfied.

Our approach to the studied problem is to relate this work to the so-called inverse procedure, a pole assignment procedure [2]. This link makes possible to solve the problem stated above. Consider the time-invariant system given by (2.1) and, without loss of generality (see Remark 3.3), assume that matrix $A$ has $(n-m)$ stable eigenvalues. Resolution of equation

$$
X A+X B X=H X
$$


gives a state feedback assigning spectrum of matrix $H\left(\sigma(H) \subset \mathbb{D}_{s}\right)$ in closed loop together with the stable part of spectrum of matrix $A$. For this equation to have a valid solution, the eigenvectors $\zeta_{i}$ of matrix $H$ must satisfy the following conditions:

$$
\begin{gathered}
\sigma(H) \cap \sigma(A)=\varnothing, \\
B \zeta_{i} \neq 0, \quad i=1, \ldots, m, \\
\zeta_{i}, \quad i=1, \ldots, m, \text { are linearly independent. }
\end{gathered}
$$

There exits a unique solution to (3.6) if and only if

$$
\left\{\begin{array}{lll}
\chi_{1} & \cdots & \chi_{n}
\end{array}\right\} \text { are linearly independent, }
$$

where $\chi_{i}, i=m+1, \ldots, n$, are eigenvectors associated to the stable eigenvalues of matrix $A$, and $\chi_{i}, i=1, \ldots, m$, can be computed by

$$
\chi_{i}=\left(\lambda_{i} I_{n}-A\right)^{-1} B \zeta_{i}, \quad i=1, \ldots, m .
$$

Hence, the stabilizing state feedback gain that solves the problem is given by

$$
F=\left[\begin{array}{llllll}
\zeta_{1} & \cdots & \zeta_{m} & 0 & \cdots & 0
\end{array}\right]\left[\begin{array}{llllll}
\chi_{1} & \cdots & \chi_{m} & \chi_{m+1} & \cdots & \chi_{n}
\end{array}\right]^{-1} .
$$

Remark 3.3. Without loss of generality, it was assumed that the system possesses $(n-m)$ stable eigenvalues. If this is not true, it is always possible to augment the representation as follows: let $v \in \mathbb{R}$ be a vector of fictitious inputs such that

$$
-v_{\min } \leq v \leq v_{\max }-\Delta_{\min }^{v} \leq \delta v \leq \Delta_{\max }^{v}
$$

where $v_{\min }, \Delta_{\min }^{v}, v_{\max }$, and $\Delta_{\max }^{v}$ are freely chosen constraints. In this case, vectors $U$ and $\Delta$ become

$$
U=\left[\begin{array}{c}
u_{\max } \\
v_{\max } \\
u_{\min } \\
v_{\min }
\end{array}\right], \quad \Delta=\left[\begin{array}{c}
\Delta_{\max } \\
\Delta_{\max }^{v} \\
\Delta_{\min } \\
\Delta_{\min }^{v}
\end{array}\right] .
$$

The augmented system is then given by

$$
\delta x(\cdot)=A x(\cdot)+\left[\begin{array}{ll}
B & 0
\end{array}\right]\left[\begin{array}{l}
u(\cdot) \\
v(\cdot)
\end{array}\right] .
$$

It is easy to see that for the obtained square system, the problem of $(n-m)$ stable eigenvalues is eliminated and controllability is not changed.

\section{Main results}

Let us now extend the previous results to the case of systems with additive bounded disturbances. To do so, a technical lemma that was established in [23] is revisited in the 
6 Constrained control and rate with disturbances

case of bounded disturbances. Relating that lemma to the pole assignment procedure presented earlier enables to find stabilizing controllers for systems with nonsymmetrical constrained control and rate or increment.

LEMma 4.1. The evolution of the autonomous system (3.1) respects rate or increment constraints if and only if matrix $H$ satisfies

$$
\begin{aligned}
& \tilde{H} Z+\widetilde{E} P \leq \Delta \quad \text { for the continuous-time case, } \\
& \widetilde{(H-\square)} Z+\widetilde{E} P \leq \Delta \text { for the discrete-time case, }
\end{aligned}
$$

where

$$
Z=\left[\begin{array}{c}
z_{\max } \\
z_{\min }
\end{array}\right], \quad \Delta=\left[\begin{array}{c}
\Delta_{\max } \\
\Delta_{\min }
\end{array}\right], \quad P=\left[\begin{array}{c}
p_{\max } \\
p_{\min }
\end{array}\right] .
$$

Proof. Let us begin by the continuous-time case.

If part. Assume that condition (4.1) is satisfied, then,

$$
-z_{\min } \leq z(k) \leq z_{\max }
$$

next, decompose matrix $H=H^{+}-H^{-}$; premultiplying (4.4) by $H^{+}$and $-H^{-}$gives

$$
\begin{gathered}
-H^{+} z_{\min } \leq H^{+} z(t) \leq H^{+} z_{\max }, \\
-H^{-} z_{\max } \leq-H^{-} z(t) \leq H^{-} z_{\min } .
\end{gathered}
$$

Further, consider the bounds on the disturbance $p(t)$ :

$$
-p_{\min } \leq p(t) \leq p_{\max }
$$

Applying to (4.6) the same technics used to achieve (4.5) where matrix $E=E^{+}-E^{-}$leads to the following inequalities:

$$
\begin{gathered}
-E^{+} p_{\min } \leq E^{+} p(t) \leq E^{+} p_{\max }, \\
-E^{-} p_{\max } \leq-E^{-} p(t) \leq E^{-} p_{\min } .
\end{gathered}
$$

Addition of the inequalities (4.5) and (4.7) enables us to write the following:

$$
\begin{aligned}
& -E^{+} p_{\min }-E^{-} p_{\max }-H^{+} z_{\min }-H^{-} z_{\max } \\
& \quad \leq H z(t)+E p(t) \leq H^{+} z_{\max }+H^{-} z_{\min }+E^{+} p_{\max }+E^{-} p_{\min }
\end{aligned}
$$

according to condition (4.1), this is equivalent to

$$
-\Delta_{\min } \leq \dot{z}(t) \leq \Delta_{\max }
$$

Only if part. Now, we assume that the derivative of $z(t)$ respects the constraints, and we suppose that condition (4.1) was not satisfied for an index $1 \leq i \leq n$ such that

$$
[\tilde{H} Z]_{i}+[\widetilde{E} P]_{i}>\Delta_{i}
$$


expanding (4.10),

$$
\left[H^{+} z_{\max }+H^{-} z_{\min }\right]_{i}+\left[E^{+} p_{\max }+E^{-} p_{\min }\right]_{i}>\Delta_{\max }^{i} \cdot
$$

Then, the following state vector for the system can be selected:

$$
\phi(t)= \begin{cases}z_{\max }^{j} & \text { if } h_{i j}>0, \\ 0 & \text { if } h_{i j}=0, j=1, \ldots, n, \\ -z_{\min }^{j} & \text { if } h_{i j}<0 .\end{cases}
$$

It is easy to check that $\phi(t)$ is an admissible state for the system. Further, the following admissible perturbation may occur:

$$
\kappa(t)= \begin{cases}p_{\max }^{j} & \text { if } e_{i j}>0, \\ 0 & \text { if } e_{i j}=0, j=1, \ldots, p, \\ -p_{\min }^{j} & \text { if } e_{i j}<0 .\end{cases}
$$

Calculation of the $i$ th component of the derivative of this state gives

$$
\begin{aligned}
{\left[\frac{d}{d t} \phi(t)\right]_{i} } & =[H \phi(t)+E p(t)]_{i}=\sum_{j=1}^{n} h_{i j} \phi_{j}(t)+\sum_{j=1}^{p} e_{i j} \kappa_{j}(t) \\
& =\left[H^{+} z_{\max }+H^{-} z_{\min }+E^{+} p_{\max }+E^{-} p_{\min }\right]_{i} .
\end{aligned}
$$

Taking into account inequality (4.10), it is possible to write

$$
\left[\frac{d}{d t} \phi(t)\right]_{i}>\Delta_{\max }^{i}
$$

which contradicts the assumption that teh derivative respects the constraints.

For the discrete-time case, assume that condition (4.2) holds true and write the increment as

$$
\begin{aligned}
z(k+1)-z(k) & =H z(k)+E p(k)-z(k) \\
& =(H-\square) z(k)+E p(k) \\
& =G z(k)+E p(k) .
\end{aligned}
$$

Making the same reasoning as the continuous-time case with matrix $H$ replaced by $G$, the proof of the if part may be easily deduced.

The necessary part is also deduced by replacing matrix $H$ by matrix $G$ and the derivative of the state $\phi(t)$ by the increment $\phi(t+1)-\phi(t)$.

Now, it is possible to derive the following lemma. 
8 Constrained control and rate with disturbances

Lemma 4.2. Domain (3.2) is $D_{P}$-positively invariant with respect to motion of system (3.1) and rate or increment constraints (3.4) are respected if and only if

$$
\begin{gathered}
\tilde{H} Z+\widetilde{E} P \leq \Delta \text { for the continuous-time case, } \\
\tilde{H}_{c} Z+\widetilde{E} P \leq 0 \quad \text { for the continuous-time case, } \\
\widetilde{(H-\square)} Z+\widetilde{E} P \leq \Delta \text { for the discrete-time case, } \\
\tilde{H} Z+\widetilde{E} P \leq Z \text { for the discrete-time case. }
\end{gathered}
$$

Proof. For the rate constraints, conditions (4.17), (4.19) can be derived from Lemma 4.1, and the $D_{P}$-positive invariance conditions (4.18), (4.20) are recalled in Theorem $3.2[4$, $8,22]$.

With this background, we are now able to solve the problem stated in Section 2. Consider the linear time-invariant stabilizable system with additive disturbances and constraints on both control and rate of the control (2.1)-(2.4). Using the state feedback

$$
u(t)=F x(t), \quad F \in \mathbb{R}^{m \times n}, \quad \sigma(A+B F) \in \mathbb{D}_{s}
$$

induces the following domain of linear behaviour in the state space:

$$
D_{F}=\left\{x \in \mathbb{R}^{n},-u_{\min } \leq F x \leq u_{\max }, u_{\min }, u_{\max } \in \operatorname{Int} \mathbb{R}_{+}^{m}\right\}
$$

If the state does not leave the domain (4.22), the control signal does not violate the constraints. That is, the set $D_{F}$ is $D_{P}$-positively invariant with respect to motion of system (2.1). This gives the following result.

Proposition 4.3. The disturbed system (2.1) with state feedback (4.21) is asymptotically stable at the origin from all initial states $x_{o} \in D_{F}$, respecting constraints on both the control and its rate (or increment), if there exists a matrix $H \in \mathbb{R}^{m \times m}$ satisfying conditions (3.7), such that

(i)

$$
F A+F B F=H F
$$

(iia)

$$
\begin{aligned}
& \tilde{H} U+\widetilde{(F E)} P \leq \Delta \\
& \tilde{H}_{c} U+\widetilde{(F E)} P \leq 0
\end{aligned} \text { for the continuous-time case, }
$$

(iib)

$$
\begin{gathered}
\widetilde{(H-\square)} U+\widetilde{(F E)} P \leq \Delta \\
\tilde{H} U+\widetilde{(F E)} P \leq U
\end{gathered}
$$


Proof. Introducing the following change of coordinates $z=F x$, it is possible to write

$$
\begin{aligned}
\delta z(t) & =F \delta x(t)=F(A+B F) x(t)+F E p(t) \\
& =H F x(t)+F E p(t)=H z(t)+F E p(t) .
\end{aligned}
$$

With this transformation, domain $D_{F}$ is transformed into domain $D_{z}$ given by (3.2). Further, with conditions (4.24), it is easy to note that domain $D_{z}$ is $D_{P}$-positively invariant with respect to the system (4.26), while the constraints on the increment of the control are respected. Bearing in mind that $\sigma(A+B F) \in \mathbb{D}_{s}$ and that the linear behaviour is guaranteed, it is possible to conclude the asymptotic stability of the closed loop system.

The steps to follow for design of such controllers are the same as those proposed in the algorithm reported in [24] for systems with nonadditive disturbances. Methods to choose the matrix $H$ for different cases were widely discussed in [24].

Comments 4.4. It is true that the set of positive invariance in this case is not the absolute maximal but it is maximal with respect to the chosen feedback $F$. In fact, for a given matrix feedback $F$, the maximal set where no violation of control constraints may occur is the set $D_{F}$ as proposed above. Nevertheless, piecewise techniques [3] or maximization procedure for the set of positive invariance [24] can be used to overcome this difficulty.

Some closed loop specifications lead to pole assignment in a specified complex plane region. In this case, the spectrum of matrix $H$ lies within a specified complex plane if conditions given in [7] are fulfilled. These conditions can easily be added as conditions on the matrix $H$.

4.1. The maximal disturbance set. As vector $Z$ and matrix $\tilde{H}$ for the continuous-time case or $(H-\square)$ and vector $Z$ have positive components, inequality (4.1) or (4.2) can never be satisfied if the difference $(\Delta-\tilde{E} P)$ was negative. Hence, the interesting conclusion is that inequality (4.1) permits to compute the maximum perturbation set that can be admissible with these rate constraints; that is, the set given by

$$
D_{P}^{\max }=\left\{p(t) \in \mathbb{R}^{p},-p_{\min } \leq p(t) \leq p_{\max } / \tilde{E} P=\Delta\right\} .
$$

Since matrix $E$ and vector $\Delta$ are known from the statement of the problem, it can be concluded if such rate constraints requirement could be fulfilled or not, and if it is admissible or not.

As stated above, the maximal disturbance set such that asymptotic stability of the closed loop system with no violation of constraints on both rate or increment and control can be estimated. Consider the system with additive disturbances

$$
\dot{x}(t)=A x(t)+B u(t)+E p(t)
$$

stabilized by state feedback (4.21). The maximal disturbance allowed set can be estimated as follows.

Corollary 4.5. The maximal disturbance set such that closed loop asymptotic stability can be ensured and rate (or increment) and magnitude control constraints are not violated is 
given by

$$
D_{P}^{\max }=\left\{p(t) \in \mathbb{R}^{p /-} p_{\min }^{\max } \leq p(t) \leq p_{\max }^{\max }\right\}
$$

where vector $P^{\max }$ satisfies

$$
\begin{aligned}
& \widetilde{F E} P^{\max }=\min \left(\Delta-\tilde{H} U,-\tilde{H}_{c} U\right) \quad \text { for the continuous-time case, } \\
& \widetilde{F E} P^{\max }=\min (\Delta-\widetilde{(H-\square)} U, U-\tilde{H} U) \text { for the discrete-time case, }
\end{aligned}
$$

the minimum here is taken componentwise.

Proof. Let us begin by the continuous-time case and assume that the objective is to stabilize the system by state feedback with $p(t)$ is a perturbation vector with unknown limits. From condition (4.24), one can write

$$
\widetilde{F E P} \leq \Delta-\tilde{H}, U \quad \widetilde{F E P} \leq-\tilde{H}_{c} U
$$

hence, the maximal disturbance set that can be seen as the limit of fulfillment of the previous two conditions. Further, for any vector $T \in \mathbb{R}^{p}$ such that $T \leq P^{\max }$, it is easy to check that condition (4.24) is satisfied.

For the discrete-time case, the proof is similar.

\section{Example}

Application of the previous results to control of DC motor for positioning of circuits boards in a surface mount robot presented in [20] is considered. The $Y$-axis of the machine uses a ball screw transmission driven by a current controlled DC motor. The rotation of the DC motor is converted into a translation motion by ball screw. A positioning table attached to the ball nut carries different loads. The process can be simplified as a two-mass system

$$
\begin{gathered}
J_{m} \ddot{\theta}_{m}+b_{m} \dot{\theta}_{m}=K_{m} i_{m}-T_{f}-T_{l}, \\
T_{l}=k_{t}\left(\theta_{m}-\frac{x_{l}}{p}\right), \\
m_{l} \ddot{x}_{l}+b_{l} \dot{x}_{l}=\frac{T_{l}}{p},
\end{gathered}
$$

where $\theta_{m}$ and $x_{l}$ are the motor angle and table displacement, respectively, $i_{m}$ is the motor current, $T_{l}$ is the load torque due to the torsion of ball screw, $T_{f}$ is the friction torque, $p$ is the screw pitch, $J_{m}$ is the motor inertia plus ball screw inertia, $b_{m}, b_{l}$, and $K_{m}$ are, respectively, the damping coefficients and the constant torque, $k_{t}$ is the stiffness, $m_{l}$ is the equivalent mass of load, table, and nut [20]. Converting the motor angle position to linear position and rewriting the model in the state space gives

$$
x \dot{(t)}=A x(t)+B u(t)-B p(t)
$$


where

$$
A=\left[\begin{array}{cc}
0 & 1 \\
0 & -9.67
\end{array}\right], \quad B=\left[\begin{array}{c}
0 \\
7.35
\end{array}\right]
$$

and $x=[p \theta p \dot{\theta}]^{t}, u(t)=i_{m}(t)$, and $p(t)$ is the lumped disturbance of load torque, friction, and other external disturbances. The current input of the DC motor, its rate, and the perturbation are, respectively, constrained as follows:

$$
-4 \leq u(t) \leq 4, \quad-80 \leq \dot{u}(t) \leq 80, \quad-1 \leq p(t) \leq 1 .
$$

Comments 5.1. The origin of the rate constraints is that the limit of the variation of the motor current $i_{m}(t)$ is taken as a peak-to-peak value at a sampling time. As the system possesses 1 stable eigenvalue, in this case, it is not necessary to augment the system. Further, for this system, the constraints are symmetric from the original example. This fact simplifies a number of conditions but we insist to present the theoretical nonsymmetrical case for the sake of generality. Furthermore, the resulting closed loop cannot be unstable as it is our goal to stabilize the system. Hence, assuming the perturbation is limited within the given set is always true although it is a function of the state of the system as presented.

Matrix $H$ reduces to a scalar. In this case, let us select $h=-\alpha$, where $\alpha \neq 0$ is any positive number which satisfies all the required conditions (3.7),

$$
\tilde{H}_{c} U=\left[\begin{array}{ll}
-4 \alpha & -4 \alpha
\end{array}\right]^{t} \leq 0
$$

It is clear that $\alpha$ here may be any positive number. To show the importance of conditions including the disturbances, we begin by the case where such conditions for the disturbed system are not satisfied. Hence, let $h=-\alpha=-20$, then the stabilizing gain feedback is given by

$$
F=\left[\begin{array}{ll}
-26.3129 & -2.7211
\end{array}\right]
$$

It is easy to check that one of the two conditions is not satisfied, that is,

$$
\begin{gathered}
\tilde{H} U+\widetilde{(-F B)} P \leq \Delta, \\
{\left[\begin{array}{l}
4 \alpha \\
4 \alpha
\end{array}\right]+\left[\begin{array}{l}
20 p_{\text {min }} \\
20 p_{\text {max }}
\end{array}\right]>\left[\begin{array}{l}
\Delta_{\text {max }} \\
\Delta_{\text {min }}
\end{array}\right] .}
\end{gathered}
$$

Figures 5.1, 5.2, 5.3, and 5.4 show that in fact, the system motion does not converge to the origin, especially state $x_{1}(t)$ is divergent. We show also the control evolution which do not respect the constraints. It must be pointed out that although the initial state is chosen inside the set of linear behaviour, the system motion does not converge to the origin. 


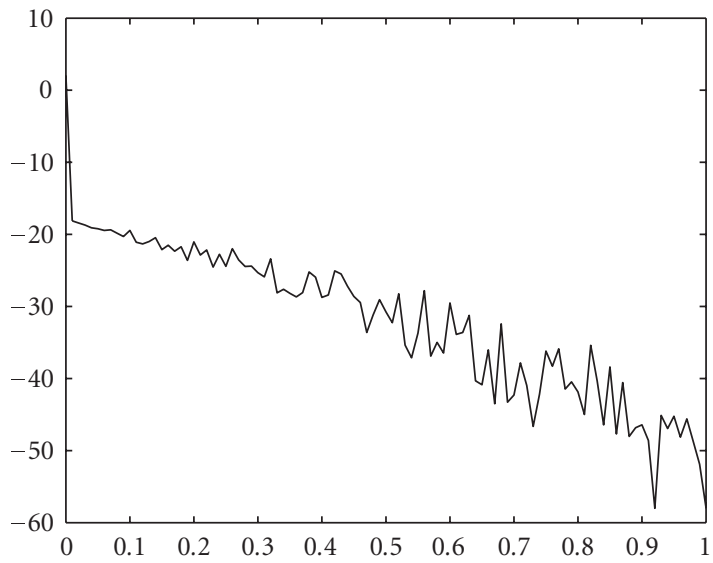

(a)



(b)

Figure 5.1. States $x_{1}(t)$ and $u(t)$ motions from the initial condition $x_{o}(t)=\left[\begin{array}{ll}2-20\end{array}\right]^{t}$.

Let us now choose $\alpha=12$. Solution of (3.6) leads to the following gain matrix $F$ :

$$
F=\left[\begin{array}{ll}
-15.7878 & -1.6327
\end{array}\right]
$$

At this step, one has to check that all required conditions (4.24) are fulfilled. In fact,

$$
\begin{gathered}
\tilde{H} U+\widetilde{(-F B) P} \leq \Delta, \\
{\left[\begin{array}{l}
4 \alpha \\
4 \alpha
\end{array}\right]+\left[\begin{array}{l}
12 p_{\min } \\
12 p_{\max }
\end{array}\right] \leq\left[\begin{array}{c}
\Delta_{\max } \\
\Delta_{\min }
\end{array}\right],}
\end{gathered}
$$




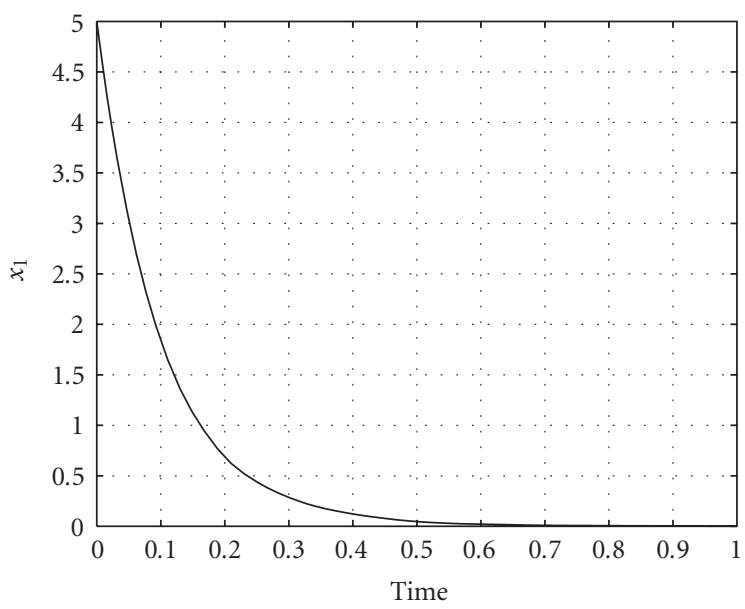

(a)

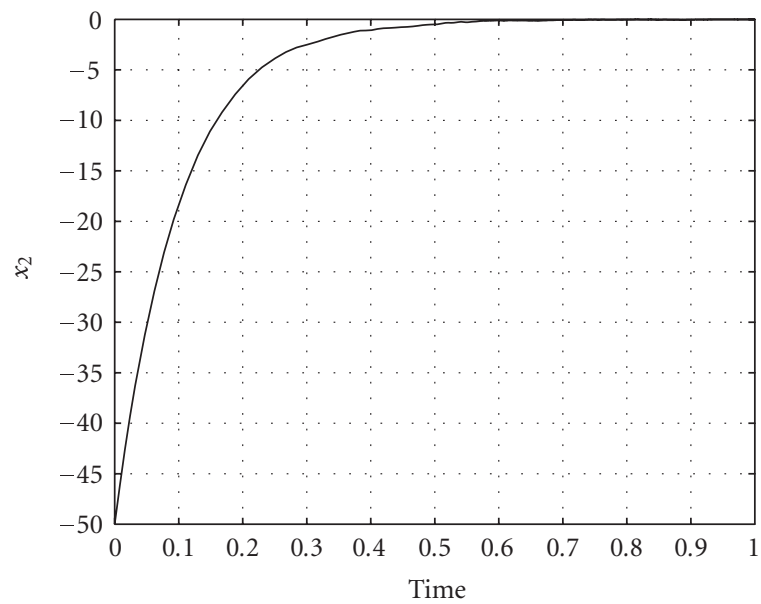

(b)

Figure 5.2. States $x_{1}(t)$ and $x_{2}(t)$ motions from the initial condition $x_{o}(t)=[5-50]^{t}$.

that is, $4 \alpha+12 p_{\max }=60 \leq 80$ which is satisfied. Then,

$$
\begin{gathered}
\tilde{H}_{c} U+\widetilde{(-F B)} P \leq 0, \\
{\left[\begin{array}{l}
-4 \alpha \\
-4 \alpha
\end{array}\right]+\left[\begin{array}{l}
12 p_{\max } \\
12 p_{\min }
\end{array}\right]=\left[\begin{array}{l}
-36 \\
-36
\end{array}\right] \leq 0 .}
\end{gathered}
$$

Finally, all required conditions are fulfilled, asymptotic stability is obtained with the obtained state feedback. Simulation results are summarized in Figures 5.1-5.4. 


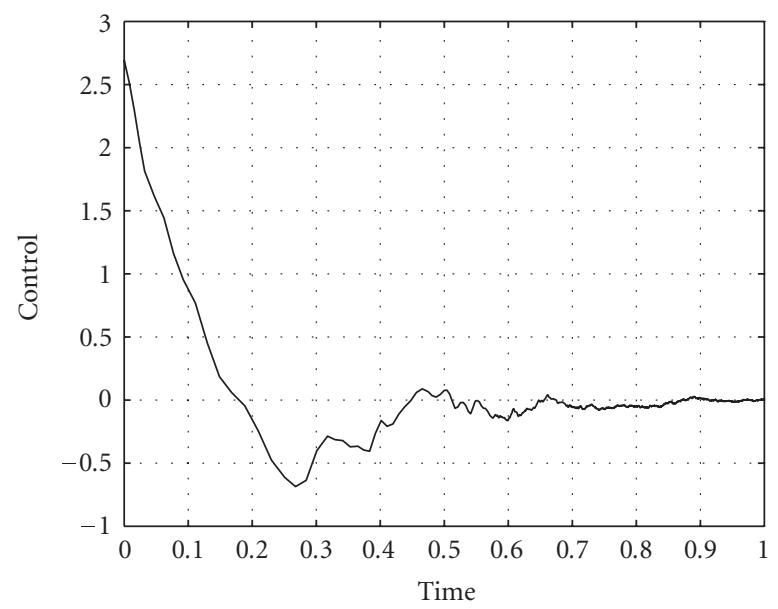

(a)

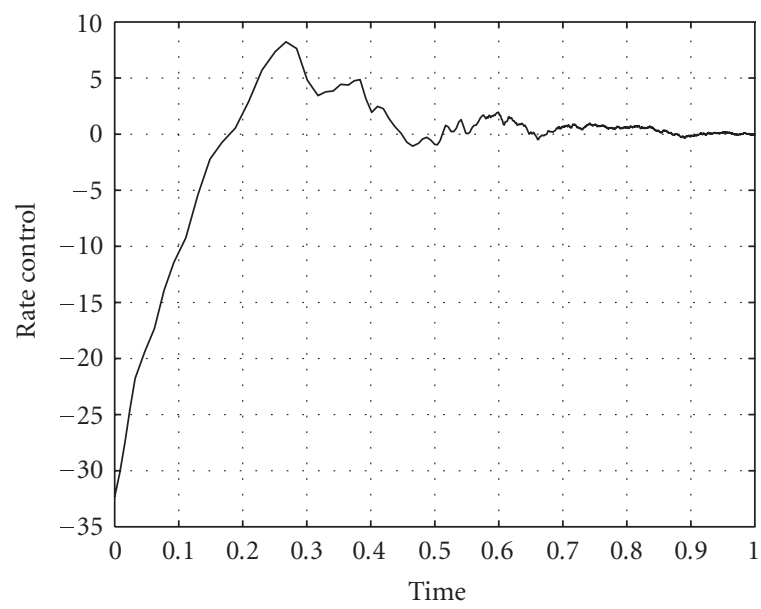

(b)

Figure 5.3. Input and rate's input evolution in time.

The maximal allowed disturbance set, which does not change stability and does not violate imposed constraints, in this case, can be easily deduced as follows:

$$
\widetilde{(-F B)} P^{\max }=\min \left(\Delta,-\widetilde{H_{c}} U\right)
$$

simple calculation leads to

$$
P^{\max }=\left[\begin{array}{l}
4 \\
4
\end{array}\right] .
$$

An example of the perturbation evolution in this case is given in Figure 5.4. 


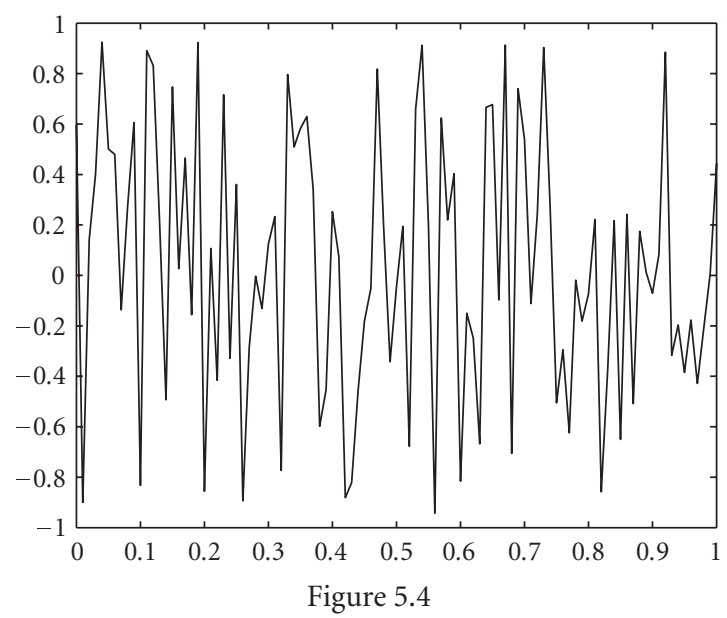

\section{Conclusion}

In this paper, the regulator problem for linear systems with additive bounded disturbances and nonsymmetrical constrained control, and rate or increment has been studied. Necessary and sufficient conditions, established for linear autonomous systems such that their motion respects rate constraints together with $D_{P}$-positive invariance, are the key to solve this problem. These conditions linked to the inverse procedure, a pole assignment method for constrained control, are the cornerstone of this work. In fact, this link enables to give a simple algorithm to compute a robust stabilizing state feedback respecting nonsymmetrical constraints on both control and rate. The maximal disturbance, such that robust asymptotic stability, rate, and control constraints are not destroyed, has also been easily deduced. An application to a positioning table has been successfully achieved, showing that the algorithm to find robust stabilizing controller is quite simple to apply.

\section{Acknowledgment}

An earlier version, limited to continuous-time case systems, of this work was presented at the ACC '04, Boston, USA.

\section{References}

[1] D. Angeli, A. Casavola, and E. Mosca, Predictive PI-control of linear plants under positional and incremental input saturations, Automatica 36 (2000), no. 10, 1505-1516.

[2] A. Benzaouia, The resolution of equation $X A+X B X=H X$ and the pole assignment problem, IEEE Transactions on Automatic Control 39 (1994), no. 10, 2091-2095.

[3] A. Benzaouia and A. Baddou, Piecewise linear constrained control for continuous-time systems, IEEE Transactions on Automatic Control 44 (1999), no. 7, 1477-1481.

[4] A. Benzaouia and C. Burgat, Regulator problem for linear discrete-time systems with nonsymmetrical constrained control, International Journal of Control 48 (1988), no. 6, 2441-2451.

[5] A. Benzaouia and A. Hmamed, Regulator problem for linear continuous-time systems with nonsymmetrical constrained control, IEEE Transactions on Automatic Control 38 (1993), no. 10, $1556-1560$. 
[6] A. Benzaouia and F. Mesquine, Regulator problem for uncertain linear discrete-time systems with constrained control, International Journal of Robust and Nonlinear Control 4 (1994), no. 3, 387395.

[7] A. Benzaouia, F. Mesquine, M. Naib, and A. Hmamed, Robust pole assignment in complex plane regions for linear uncertain constrained control systems, International Journal of Systems Science 32 (2001), no. 1, 83-89.

[8] F. Blanchini, Feedback control for linear time-invariant systems with state and control bounds in the presence of disturbances, IEEE Transactions on Automatic Control 35 (1990), no. 11, 1231-1234.

[9] __ Ultimate boundedness control for uncertain discrete-time systems via set-induced Lyapunov functions, IEEE Transactions on Automatic Control 39 (1994), no. 2, 428-433.

[10] Set invariance in control, Automatica 35 (1999), no. 11, 1747-1767.

[11] D. W. Clarke, C. Mohtadi, and P. S. Tuffs, Generalised predictive control, part 1: the basic algorithm; part 2: extension and interpretations, Automatica 23 (1987), no. 2, 137-160.

[12] M. A. Dahleh and I. J. Diaz-Bobillo, Control of a Uncertain Systems: A Linear Programming Approach, Prentice Hall, New Jersey, 1995.

[13] E. G. Gilbert and K. T. Tan, Linear systems with state and control constraints: the theory and application of maximal output admissible sets, IEEE Transactions on Automatic Control 36 (1991), no. 9, 1008-1020.

[14] D. Henrion, S. Tarbouriech, and V. Kučera, Control of linear systems subject to input constraints: a polynomial approach, Automatica 37 (2001), no. 4, 597-604.

[15] H. Hindi and S. Boyd, Analysis of linear systems with saturating control using convex optimisation, Proceedings of the 37th IEEE Conference on Decision and Control, Florida, December 1998.

[16] T. Hu and Z. Lin, The equivalence of several set invariance conditions under saturations, Proceedings of the 41st IEEE Conference on Decision and Control, Nevada, December 2002.

[17] T. Hu, Z. Lin, and B. M. Chen, Analysis and design for discrete-time linear systems subject to actuator saturation, Systems \& Control Letters 45 (2002), no. 2, 97-112.

[18] __ Analysis and design method for linear systems subject to actuator saturation and disturbance, Automatica 38 (2002), no. 2, 351-359.

[19] H. Khalil, Nonlinear Systems, Prentice Hall, New Jersey, 1996.

[20] Y. F. Li and J. Wikander, Discrete time sliding mode control of a DC motor and ball screw driven positionning table, Proceedings of the 15th IFAC World Congress, Barcelona, July 2002.

[21] Z. Lin and A. Saberi, Semi-global exponential stabilization of linear systems subject to "input saturation" via linear feedbacks, Systems \& Control Letters 21 (1993), no. 3, 225-239.

[22] F. Mesquine and D. Mehdi, Constrained observer based controller for linear continuous time systems, International Journal of Systems Science 27 (1996), no. 12, 1363-1369.

[23] F. Mesquine, F. Tadeo, and A. Benzaouia, Regulator problem for linear systems with constrained control and its increment, Proceedings of the 15th IFAC World Congress, Barcelona, July 2002.

[24] - Regulator problem for linear systems with constraints on control and its increment or rate, Automatica 40 (2004), no. 8, 1387-1395.

[25] A. A. Stoorvogel, A. Saberi, and P. Sannuti, Performance with regulation constraints, Automatica 36 (2000), no. 10, 1443-1456.

Fouad Mesquine: Equipe d'Automatique et de Contrôle des Procédés Industriels, Département de Physique, Faculté des Sciences, UCAM, BP 2390, Marrakesh, Morocco E-mail address: mesquine@ucam.ac.ma

Fernando Tadeo: Departamento de Ingenieria de Sistemas Y Automática, Universidad de Valladolid, 47005 Valladolid, Spain

E-mail address: fernando@autom.uva.es

Abdellah Benzaouia: Equipe d'Automatique et de Contrôle des Procédés Industriels, Département de Physique, Faculté des Sciences, UCAM, BP 2390, Marrakesh, Morocco E-mail address: benzaouia@ucam.ac.ma 


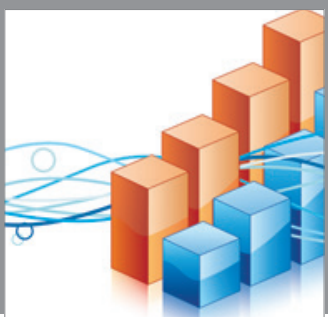

Advances in

Operations Research

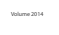

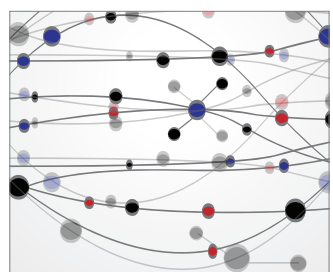

\section{The Scientific} World Journal
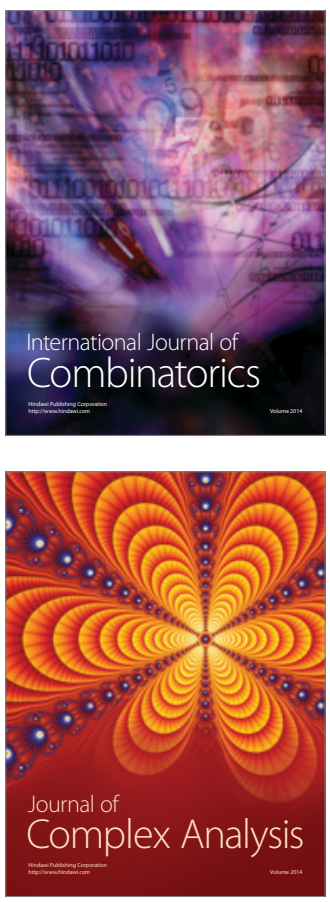

International Journal of

Mathematics and

Mathematical

Sciences
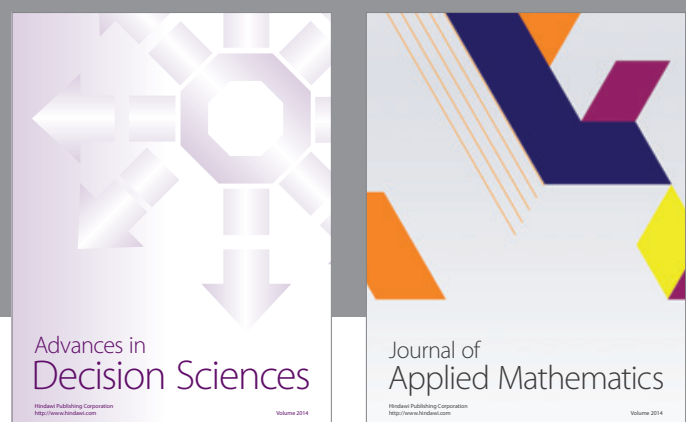

Journal of

Applied Mathematics


Submit your manuscripts at http://www.hindawi.com


Mathematical Problems in Engineering
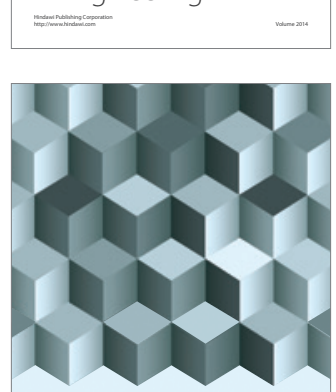

Journal of

Function Spaces
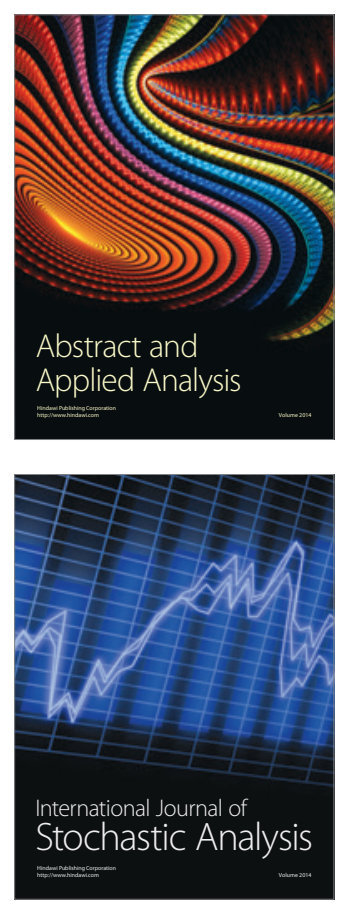

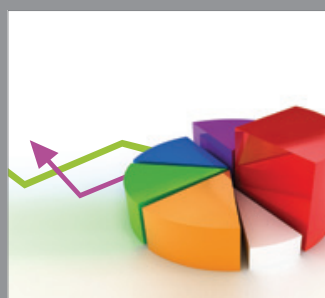

ournal of

Probability and Statistics

Promensencen
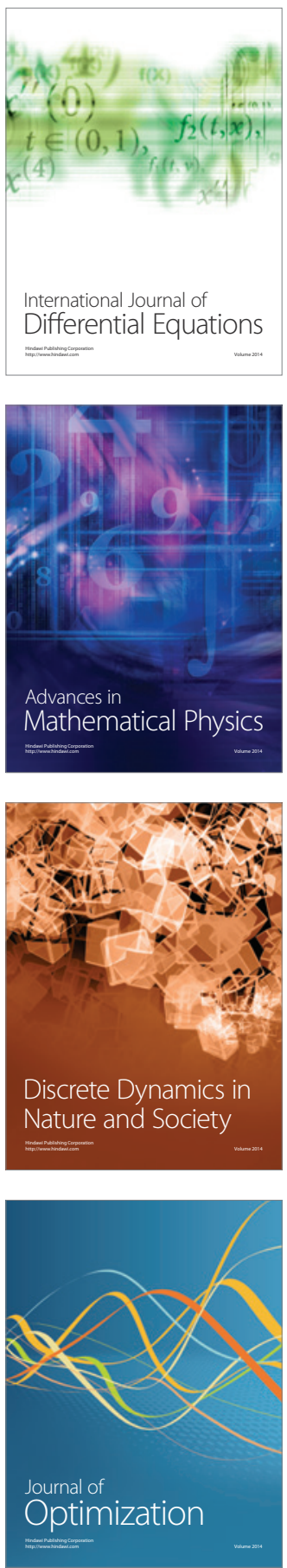